\section{RISK OF ROAD TRAFFIC INJURIES IN BARCELONA: BASELINE RESULTS FROM THE LESIONAT COHORT STUDY}

C Martin, D Prieto-Alhambra*, E Vilella, J Bel, E Martorell, M Birules, R Casas, $S$ Valiente, J L Del Val, E Briones Correspondence: Medicine Department, Institut Catala de la Salut; IDIAP Jordi Gol; Passeig Sant Joan, 20, baixos. 08015 Barcelona (Spain)

10.1136/ip.2010.029215.551

Aim To assess the relationship between medical risk factors, and traffic collisions (TC) traffic injuries (TI) the year before recruitment.

Methods Design: prospective cohorts.

Setting 26 primary care practices (PC). Urban area. Barcelona (Spain).

Population 1938 subjects possessing driving license, who attended one of the participating practices from March to November/2009.

Measurements -outcome TC, TI the year before.

Main exposures Risk-associated long-term conditions (LTC), medications, alcohol, psychoactive substance (PS)

Covariates Age, gender, driver's characteristics, social class. Data collection Structured survey (checked with medical records) during the recruitment visit.

Statistics Mann-Whitney-U to assess differences in TC and TI occurrence between categories. Logistic regression to assess relationship between TC, TI and drivers characteristics.

Results Mean age 44 years (SD 18.7). 57.3\% males. TC incidence the year before $12.6 \%$; TI $6.1 \% .51 .1 \%$ had a history of LTC, and $45.2 \%$ were taking medicines. $2.7 \%$ were risk alcohol consumers, and 14\% PS users. TC risk was higher among those less than 50 years (OR 2.17 (1.27 to 3.7)) and those with high-risk alcohol consumption (OR 4.14 (1.7 to 10.0)). TI occurrence was higher among those less than 50 years (OR 4.8 (1.8 to 12.4)) and in lower social classes (OR 9.9 (2.0 to 23.4)).

Conclusions According to our data, preventive interventions on alcohol intake, in lower social classes and among young people, are necessary. 